\title{
MULTIPLIERS OF COMMUTATIVE BANACH ALGEBRAS
}

\author{
JU-KWEI WANG
}

1. Introduction. In this paper Banach algebras will always mean complex commutative Banach algebras, with or without a unit. The concept of multipliers of a Banach algebra was introduced by Helgason [5] as follows: Let $A$ be a semisimple Banach algebra considered as an algebra of continuous functions over its regular maximal ideal space $X$. Then by a multiplier of $A$ is meant a function $g$ over $X$ such that $g A \subset A$. Every multiplier turns out to be a bounded continuous function, and the set of all multipliers of $A$ under pointwise operations forms an algebra $M(A)$, called the multiplier algebra of $A$. In particular, if $A$ is the algebra of all continuous functions on a locally compact Hausdorff space $X$ which vanish at infinity under pointwise operations and supremum norm, then $M(A)$ is the algebra of all bounded continuous functions on $X$. In this case, Buck [2] introduced a topology on $M(A)$, called the strict topology, with many nice properties. In $\S 3$, we will see that certain of Buck's results can be generalized to an arbitrary semisimple Banach algebra $A$.

The multiplier algebra can also be defined for a more general Banach algebra, and the strict topology can also be introduced in such a general case. $\S 2$ will be devoted to discussions in such generality.

Next we narrow down to the case where $A$ is a supremum norm algebra. In this case there are three natural topologies on $M(A)$, viz. the norm topology, the strict topology and the topology of uniform convergence on compact subsets of the maximal ideal space of $A$. It seems natural to ask when do two of these three topologies coincide. In $\S 4$ we seek such characterizations in terms of topological properties of the Šilov boundary of $A$. Other problems regarding supremum norm algebras will be discussed in $\S 5$.

Finally we will identify the multiplier algebras of certain Banach algebras which arise in harmonic analysis. Let $S$ be an additive semigroup of positive integers and let $A$ be the algebra of all continuous functions on the unit circle whose Fourier series involve $e^{i n \cdot x}$ with $n \in S$ only. Let $M(S)$ be the set of all integers $m$ such that $m+S \subset S$. By an application of Fejér's theorem on the Cesáro summability we can prove that $M(A)$ is the algebra of all continuous functions on the unit circle whose Fourier series involve $e^{i n x}$ with $m \in M(S)$ only. In $\S 6$ we will get a generalization of this result to arbitrary compact and even

Received June 15, 1960. This work was supported in part by the Air Force Office of Scientific Research. 
locally compact groups.

Standard terms as those given in Loomis [8] will be used. Several unsolved problems will be listed in $\$ 7$.

It is Professor H. L. Royden who aroused my interest in Banach algebras and it is Professor Karel deLeeuw who guided me through this thesis. I wish to express my cordial thanks to them both. Their influence on me will certainly remain far beyond this thesis.

2. Definition of the multiplier algebra. Let $A$ be a complex commutative Banach algebra with or without a unit. By an absolute zerodivisor of $A$ we mean an element $f \in A$ such that $f A=\{0\}$. The set of all absolute zero-divisors of $A$ is evidently a closed ideal contained in the radical of $A$. It will be called the order ideal of $A . A$ is called without order if its order ideal contains the element 0 only. Obviously $A$ is without order if either $A$ has a unit or $A$ is semisimple.

Let $A$ be a Banach algebra without order. By a multiplier of $A$ is meant a mapping $T: A \rightarrow A$ such that

$$
(T f) g=f(T g)
$$

whenever $f, g \in A$.

THEOREM 2.1. Every multiplier $T$ on a commutative Banach algebra $A$ without order is a bounded linear operator on the Banach space underlying $A$.

Proof. Let $f, g, h$ be arbitrary elements of $A$ and let $\lambda$ and $\mu$ be arbitrary scalars. Then

$$
\begin{aligned}
h \cdot T(\lambda f+\mu g) & =(\lambda f+\mu g) T h=\lambda f T h+\mu g T h \\
& =\lambda h T f+\mu h T g=(\lambda T f+\mu T g) \cdot h .
\end{aligned}
$$

Since $A$ is without order, we see that $T(\lambda f+\mu g)=\lambda T f+\mu T g$. Hence $T$ is linear.

To show that $T$ is bounded we use the closed graph theorem. Thus suppose that $f_{n}, f, g$ are elements in $A, n=1,2,3, \cdots$, such that $\lim f_{n}=f$ and $\lim T f_{n}=g$. Then for each $h \in A$ we have

$$
\begin{aligned}
g h=\left(\lim T f_{n}\right) h & =\lim \left(T f_{n} \cdot h\right)=\lim \left(f_{n} \cdot T h\right) \\
& =\left(\lim f_{n}\right) T h=f T h=h T f .
\end{aligned}
$$

Thus $g=T f$ because $A$ is without order. This shows that $T$ is bounded. q.e.d.

Note that the proof holds for any commutative topological algebra without order in which multiplication is separately continuous and for which the closed graph theorem holds. 
Now consider the set $M(A)$ of all multipliers on $A$. For each $f \in A$, define the mapping $T_{f}$ by $T_{f} g=f g$ for all $g \in A$. Evidently $T_{f}$ is a multiplier. Identifying $f$ with $T_{f}$, we get an embedding of $A$ in $M(A)$. If $A$ has a unit, then $A=M(A)$ under this identification. Hence the case where $A$ has no unit seems to be more interesting.

The multipliers $T$ on a Banach algebra $A$ without order are characterized by the condition

$$
(T f) g=T(f g), \quad f, g \in A .
$$

It is evident that every mapping satisfying this condition is a multiplier. Conversely suppose that $T$ is a multiplier. Then for all $f, g, h \in A$ we have

$$
T(f g) \cdot h=f g T h=g \cdot f T h=(g T f) h .
$$

Since $A$ is without order, we get $T(f g)=(T f) g$. Thus every multiplier satisfies this condition.

Now let $T_{1}$ and $T_{2}$ be two multipliers on $A$ and let $f, g$ be two elements of $A$. Then

$$
\begin{aligned}
f\left(T_{1} T_{2}\right) g & =\left(T_{1} f\right)\left(T_{2} g\right)=T_{2}\left(g T_{1} f\right)=T_{2} T_{1}(f g) \\
& =\left(T_{2} f\right)\left(T_{1} g\right)=g T_{1} T_{2} f .
\end{aligned}
$$

Hence $T_{1} T_{2}$ is also a multiplier; further it satisfies

$$
f\left(T_{1} T_{2} g\right)=T_{2} T_{1}(f g) .
$$

But as we remarked before,

$$
f\left(T_{1} T_{2} g\right)=T_{1} T_{2}(f g) .
$$

Hence $T_{1} T_{2}(f g)=T_{2} T_{1}(f g)$, or $f T_{1} T_{2} g=f T_{2} T_{1} g$. As $A$ is without order, it gives $T_{1} T_{2}=T_{2} T_{1}$.

Also it is evident that the identity operator is a multiplier.

Summarizing, we get the following theorem:

THEOREM 2.2. For any commutative Banach algebra $A$ without order, $M(A)$ is a commutative operator algebra with unit including an isomorphic image of $A$ as an ideal.

The algebra $M(A)$ will be called the multiplier algebra of $A$. It is so far not yet topologized. A suitable topology for it is the strong operator topology, as the following theorem shows:

Theorem 2.3. Let $A$ be a commutative Banach algebra without order and let $M(A)$ be its multiplier algebra. Then $M(A)$ is complete under the strong operator topology. 
Proof. Let $T_{a}$ be a Cauchy net in $M(A)$ with respect to the strong operator topology. Then for each $f \in A$ we have

$$
\lim _{\alpha, \beta}\left\|T_{\alpha} f-T_{\beta} f\right\|=0 .
$$

As $A$ is complete, there exists an element $T f \in A$ such that

$$
\lim T_{\alpha} f=T f .
$$

Now we show that $T$ is a multiplier. For this, take $f, g \in A$. Then

$$
g T f=g \lim T_{\alpha} f=\lim g T_{\alpha} f=\lim f T_{\alpha} g=f \lim T_{\alpha} g=f T g .
$$

Hence $T$ is a multiplier. By our definition, $T$ is the strong limit of the net $T_{\alpha}$. Hence we proved our theorem.

Thus $M(A)$ is a strongly closed operator algebra over $A$. Following Buck [2], the strong topology on $M(A)$ will also be called the strict topology. It is the locally convex topology defined by the seminorms $\|T\|_{f}=\|T f\|$, where $f$ runs over $A$.

As $M(A)$ is strongly closed, it is also uniformly closed. Since $A \subset M(A)$, elements $x$ in $A$ also have operator norms

$$
\text { || } x\|\|=\sup _{|| y||=1}\|x y\| \text {. }
$$

With respect to the operator norm, $A$ is a normed algebra. Later we will give an example showing that $A$ is not necessarily complete with respect to the operator norm $|\|\cdot \mid\|$. Evidently $A$ is complete with respect to $\||\cdot|\|$ if and only if $\||\cdot|\|$ and $\|\cdot\|$ are equivalent.

Let $A$ be a Banach algebra, then a net $\left\{f_{\alpha}\right\}$ in $A$ is called an approximate unit if $f_{\alpha} f \rightarrow f$ for all $f \in A$. Many algebras in harmonic analysis have approximate units. It is easy to show

Theorem 2.4. A Banach algebra $A$ without order is strictly dense in its multiplier algebra $M(A)$ if and only if $A$ has an approximate unit.

The following observation, due to Devinatz and Hirschman, is curious:

THEOREM 2.5. If $A$ is a Banach algebra without order and if $T: A \rightarrow A$ is a bijective (i.e., one-to-one and onto) multiplier, then $T^{-1}$ is also a multiplier.

Proof. $\left(T^{-1} f\right) g=T^{-1} T\left[\left(T^{-1} f\right) g\right]=T^{-1}\left[\left(T T^{-1} f\right) g\right]=T^{-1}(f g)$. q.e.d.

Thus the set of all bijective multipliers coincides with the set of all the multipliers invertible in the multiplier algebra, and the spectrum 
of an element in $M(A)$ remains the same when $M(A)$ is extended to the full non-commutative algebra of bounded linear operators in $A$.

3. Multipliers of semisimple algebras. Let $A$ be a semisimple Banach algebra and let $X$ be its maximal ideal space. (By the maximal ideal space of a Banach algebra without unit we mean the space of all regular maximal ideals). It is well-known that $X$ is a locally compact Hausdorff space under the weak topology and every element $f \in A$ can be considered as a continuous function on $X$ vanishing at infinity. The norm $\|f\|$ of $f$ in general exceeds its supremum norm $\|f\|_{\infty}=\sup _{x \in X}|f(x)|$ when considered as a function. When $A$ is thus realized, every multiplier of $A$ can also be realized as a bounded continuous function, as the following theorem shows:

THEorem 3.1. Let $A$ be a semisimple Banach algebra with maximal ideal space $X$ and let $T$ be a multiplier of $A$. Then there is a bounded continuous function $g$ on $X$ such that

$$
(T f)(x)=g(x) f(x)
$$

for all $f \in A$ and all $x \in X$. Further $\|g\|_{\infty} \leqq\|T\|$.

Proof. Let $x \in X$ be an arbitrary point. If $f_{1}, f_{2} \in A$ and $f_{1}(x) \neq 0$, $f_{2}(x) \neq 0$, then by the assumption that $T$ is a multiplier we have

$$
\frac{\left(T f_{1}\right)(x)}{f_{1}(x)}=\frac{\left(T f_{2}\right)(x)}{f_{2}(x)} \text {. }
$$

For each $x \in X$ select $f \in A$ with $f(x) \neq 0$. Then define

$$
g(x)=\frac{(T f)(x)}{f(x)} .
$$

The previous considerations show that this is independent of the selection of $f$ and defines a continuous function $g$ on $X$. Further

$$
(T f)(x)=g(x) f(x)
$$

holds identically even if $f(x)=0$. Hence we have only to show that $g$ is bounded and $\|g\|_{\infty} \leqq\|T\|$.

To prove this, let

$$
K_{x}=\sup _{\|f\|=1}|f(x)|
$$

for $x \in X, f \in A$. Then $0<K_{x} \leqq 1$. Thus

$$
|g(x) f(x)| \leqq K_{x}\|g f\|=K_{x}\|T f\| \leqq K_{x}\|T\|\|f\|
$$


for all $f \in A$, in particular, for all $f$ satisfying $\|f\|=1$. Therefore

$$
|g(x)| \leqq \inf _{\|f\|=1} \frac{K_{x}\|T\|}{|f(x)|}=\frac{K_{x}\|T\|}{\sup _{\|f\|=1}|f(x)|}=\|T\| .
$$

As this holds for all $x \in X$, we have

$$
\|g\|_{\infty} \leqq\|T\|,
$$

as desired.

q.e.d.

By this theorem we can identify $g$ with $T$ and consider $M(A)$ as the set $\{g: g A \subset A\}$ of complex-valued functions on $X$. Thus $M(A)$ is the largest function algebra on $X$ containing $A$ as an ideal. This was the original definition given by Helgason [5]. There he mentioned that $\mathrm{H}$. Mirkil pointed out to him that every $g \in M(A)$ is a bounded continuous function, but no explicit proof was given.

One of the advantages of the abstract definition of multipliers is that it works equally well for function algebras not necessarily over the maximal ideal space. Thus if $A$ is a separating algebra over a locally compact space $S$, e.g. the Silov boundary of $A$, then $M(A)$ is isomorphic to the set of functions $g$ on $S$ such that $g A \subset A$.

As $M(A)$ is a Banach algebra with respect to its operator norm, it has its own maximal ideal space $Y$. Let $H$ be the hull of $A$ in $Y$ and let $K=Y \backslash H$. As $A \subset M(A)$, and as the homomorphisms corresponding to the maximal ideals in $K$ do not take all of $A$ to 0 , the intersections of $A$ and the elements of $K$ are regular maximal ideals of $A$.

Suppose that $\varphi_{1}$ and $\varphi_{2}$ are homomorphisms of $M(A)$ onto the complex field with the same non-zero restrictions on $A$. Then for $g \in M(A)$ and $f \in A$ we have

$$
\varphi_{1}(f) \varphi_{1}(g)=\varphi_{1}(f g)=\varphi_{2}(f g)=\varphi_{2}(f) \varphi_{2}(g) .
$$

Select $f \in A$ such that $\varphi_{1}(f)=\varphi_{2}(f) \neq 0$. Then $\varphi_{1}(g)=\varphi_{2}(g)$. Since $g$ is arbitrary, we see that $\varphi_{1}=\varphi_{2}$. Hence no two distinct elements of $K$ can have the same intersection with $A$. On the other hand, if $\varphi$ is a homomorphism of $A$ onto the complex field, and if $f \in A$ satisfies $\varphi(f)=1$, then for each $g \in M(A)$ we can define $\varphi(g)=\varphi(g f)$ unambiguously. The mapping $\varphi$ acting on $M(A)$ is easily shown to be a homomorphism. Thus every regular maximal ideal of $A$ can be extended to a unique element in $K$. Thus $k \longleftrightarrow k \cap A$ establishes a one-to-one correspondence between $K$ and $X$.

Now we show that this correspondence is a homeomorphism. Since the topology on $K$ is induced by the elements of $M(A)$ and since these elements are automatically continuous functions on $X$, the topology on $K$ is coarser than the topology on $X$. On the other hand, since 
$A \subset M(A)$ and the topology on $X$ is induced by the functions in $A$, we see in turn that topology on $K$ is finer than that on $H$. Hence the correspondence is a homeomorphism, and $X$ is thus topologically embeddable in $Y$.

The null set of each function in $A$ over $Y$ is closed. Hence the intersection $H$ of such null sets is closed, and therefore compact, because $M(A)$ has a unit.

Summarizing, we get

THEOREM 3.2. Let $X$ and $Y$ be the maximal ideal spaces of a semisimple Banach algebra $A$ and its multiplier algebra $M(A)$ respectively, both endowed with the weak topology. Then we can write

$$
Y=H \cup K
$$

where $H$ is compact, and there exists a natural homeomorphism from $K$ onto $X$.

When $Y$ is endowed with the hull-kernel topology, $X$ is dense in $Y$.

THEOREM 3.3. Let $X$ and $Y$ be the maximal ideal spaces of a semisimple Banach algebra $A$ and its multiplier algebra $M(A)$ respectively. Then when $X$ is embedded in $Y$ as in Theorem 3.2, $X$ is dense in $Y$ with respect to the hull-kernel topology.

Proof. If $g \in$ kernel $X \subset M(A)$, then $g=0$ identically. Thus every point $y \in Y$ is in the hull of the kernel of $X$. q.e.d.

The situation is completely different with the weak topology:

ExAmple. There is a semisimple Banach algebra $A$ such that

(i) the maximal ideal space $X$ of $A$ is not dense in the maximal ideal space $Y$ of $M(A)$ with respect to the weak topology.

(ii) $A$ is a *algebra but $M(A)$ is not.

(iii) $A$ is regular but $M(A)$ is not.

Construction. Let $G$ be a locally compact abelian group and let $A$ be the $L^{1}$ group algebra of $G$. Then $M(A)$ is the algebra of all complexvalued finite regular Borel measures on $G$, with convolution as multiplication. It is well-known that $A$ is a regular semisimple *-algebra. Williamson proved in [10] that $M(A)$ is also a *-algebra if and only if $G$ is discrete. Thus in case $G$ is not discrete, $M(A)$ is not a *algebra. By an argument of Hewitt [7, Theorem 6.1], we see that $X$ is not dense in $Y$. Applying Theorem 3.3, $M(A)$ cannot be regular.

Of course if $G$ is discrete, $A$ has an identity, and $M(A)=A$. Nothing of this sort will happen. 
In $\S 2$ we introduced the operator norm for $A$. The following theorem locates roughly the closure of $A$ in $M(A)$ with respect to the operator norm:

THEOREM 3.4. Using the notation of Theorem 3.2, the closure of $A$ in $M(A)$ with respect to the operator norm is contained in kernel $H$.

Proof. Suppose that $g$ is in the closure of $A$ in $M(A)$ with respect to the operator norm. For any $\varepsilon>0$ there is an $f \in A$ such that $\||g-f|\|<\varepsilon$. Now $f(x)=0$ for every $x \in H$. Hence we have

$$
|g(x)|=|g(x)-f(x)| \leqq|||g-f|||<\varepsilon .
$$

Hence $g(x)=0$ for each $x \in H$. Thus $g \in$ kernel $H$.

q.e.d.

ExAMPLE. There is a semisimple Banach algebra $A$ with the following properties:

(i) the operator norm $\|\mid \cdot\|$ is not equivalent to the algebra norm $\|\cdot\|$ in $A$.

(ii) the closure of $A$ with respect to the operator norm in $M(A)$ is properly contained in kernel $H$.

(iii) there is a function in $M(A) \backslash A$ vanishing at infinity.

Construction. Let $\varphi(x)$ be a real-valued function on the real line such that $\varphi(x) \geqq 1$ and $\lim _{x \rightarrow \pm \infty} \varphi(x)=+\infty$. Let $A$ be the set of all continuous functions $f$ on the real line such that

$$
\|f\|=\sup _{x}|f(x) \varphi(x)|<\infty .
$$

Under pointwise operations, $A$ becomes a Banach algebra. The properties (i)-(iii) are easy to verify.

Next we consider the strict closure $B$ of $A$ in $M(A)$. As $B$ is closed with respect to the operator norm, it is also a Banach algebra.

Theorem 3.5. If $A$ is a semisimple Banach algebra and if $B$ is its strict closure in $M(A)$, then $M(A) \subset M(B)$.

If further $A$ is equal to the norm closure of $A^{2}$, then $M(A)=M(B)$.

Proof. We first prove that $M(A) \subset M(B)$. Let $g \in M(A)$ and $h \in B$. Then there is a net $f_{\alpha} \rightarrow h$ strictly, with $f_{\alpha} \in A$. Since multiplication is separately continuous with respect to the strict topology, we have $g f_{\alpha} \rightarrow g h$ strictly. As $g f_{\alpha} \in A$ for each $\alpha$, we have $g h \in B$. Hence $g \in M(B)$. This proves that $M(A) \subset M(B)$.

Now suppose that $A$ is equal to the norm closure of $A^{2}$, and suppose that $g \in M(B) ; f_{1}, f_{2} \in A$. Then $g f_{1} \in B \subset M(A)$ and thus $g f_{1} f_{2} \in A$. As 
elements of the form $f_{1} f_{2}$ generate $A, g \in M(A)$. As $g$ is arbitrary, $M(B) \subset M(A)$. This combined with the previous paragraph gives the desired result.

q.e.d.

4. The multipliers of a supremum norm algebra. A semisimple Banach algebra $A$ is called a supremum norm algebra if

$$
\|f\|=\|f\|_{\infty}=\sup _{x \in X}|f(x)|
$$

for all $f \in A$, where $X$ denotes the maximal ideal space of $A$. The multiplier algebra of a supremum algebra is specially easy to handle. To start with, we have

TheoRem 4.1. If $A$ is a supremum norm algebra, then so is $M(A)$ with its operator norm.

Proof. Take $g \in M(A)$. Let $\|g\|$ denote the operator norm of $g$. Then $\|g\|_{\infty} \leqq\|g\|$. But

$$
\|g\|=\sup _{\|f\|=1}\|g f\|_{\infty} \leqq \sup _{\|f\|=1}\|g\|_{\infty}\|f\|_{\infty}=\|g\|_{\infty} .
$$

Hence $\|g\|=\|g\|_{\infty}$.

q.e.d.

Notice that in this theorem we did not specify whether $\|g\|_{\infty}$ denotes $\sup |g(x)|$ over the maximal ideal space $X$ of $A$ or over the maximal ideal space $Y$ of $M(A)$. It holds equally true. Further, as both the supremum norm topology and the strict topology are complete, it follows from the open mapping theorem that these topologies coincide if and only if the strict topology is metrizable.

If $A$ is a supremum norm algebra, in addition to these two topologies on $M(A)$ there is another interesting topology, viz., the topology of uniform convergence on compact subsets of the maximal ideal space $X$ of $A$. Following Buck [2], we will denote the supremum norm topology, the strict topology and the topology of uniform convergence on compact subsets by $\sigma, \beta$, and $\kappa$ respectively. The topology $\kappa$ can be described by the seminorms $\|g\|_{K}=\sup _{x \in K}|g(x)|$, where $K$ runs over all compact subsets of $X$. The following theorem compares the fineness of these topologies.

THeorem 4.2. $\sigma$ is finer than $\beta$ and $\beta$ is finer than $\kappa$.

Proof. The first statement is obvious. To show that $\beta$ is finer than $\kappa$, take a set $S \subset M(A)$. Let $B$ and $C$ be the $\beta$ - and $\kappa$-closures of $S$ respectively. We have only to show that $B \subset C$. For this, take $g_{0} \in B$, and let $U$ be a $\kappa$-neighborhood of $g_{0}$ given by 


$$
U=\left\{g \in M(A):\left|g(x)-g_{0}(x)\right|<\varepsilon \text { for all } x \in K\right\},
$$

where $K$ is a fixed compact subset of $X$ and $\varepsilon>0$. For each $x_{0} \in K$ there is a function $f \in A$ such that $|f(x)|>1$ in a neighborhood of $x_{0}$. Cover $K$ with a finite number of such neighborhoods corresponding to the functions $f_{1}, f_{2}, \cdots, f_{n} \in A$. Let

$$
V=\left\{g \in M(A): \sup _{i=1,2, \cdots, n}\left\|\left(g-g_{0}\right) f_{i}\right\|<\varepsilon\right\} .
$$

The $V$ is a $\beta$-neighborhood of $g_{0}$. Since $g_{0} \in B$, we can find an element $g \in S \cap V$. But then

$$
\sup _{x \in K}\left|g(x)-g_{0}(x)\right| \leqq \sup _{i=1,2, \cdots, n}\left\|\left(g-g_{0}\right) f_{i}\right\|<\varepsilon
$$

and hence $g \in U$. Thus $g_{0} \in C$ and hence $B \subset C$.

q.e.d.

In a locally convex topological space, a set is called bounded if each seminorm defining the topology assigns a bounded set of values to it.

THEOREM 4.3. (i) In $M(A)$ every $\sigma$-bounded set is $\beta$-bounded and every $\beta$-bounded set is $\kappa$-bounded.

(ii) The $\beta$-closure and the $\kappa$-closure of a $\sigma$-bounded set in $M(A)$ coincide; in other words, the topologies $\beta$ and $\kappa$ agree on $\sigma$-bounded sets.

Proof. (i) It is evident that every $\sigma$-bounded set is also $\beta$-bounded. Let now $S$ be a $\beta$-bounded set. Take an arbitrary compact set $K \subset X$. Cover $K$ with a finite number of open sets corresponding to the functions $f_{1}, f_{2}, \cdots, f_{n} \in A$ as was described in the proof of Theorem 4.2. Then $\|g\|_{K} \leqq \sup _{i=1,2, \cdots, n}\left\|g f_{i}\right\|$ for all $g \in S$. As $S$ is $\beta$-bounded, the righthand side of the inequality is a bounded set of numbers. Hence $S$ is also $\kappa$-bounded.

(ii) Let $B$ and $C$ be the $\beta$ - and $\kappa$-closures of $S$ respectively. By 4.2, $B \subset C$. Now suppose $g \in C$ and let $\left\{g_{\alpha}\right\}$ be a net in $S \kappa$-converging to $g$. Let $f \in A$ and $\varepsilon>0$ be given. Since $f$ vanishes at infinity, there is a compact set $K \subset X$ such that $|f(x)|<\varepsilon$ for $x \notin K$. Then

$$
\begin{aligned}
\left\|g_{\alpha} f-g f\right\| & =\sup _{x \in X}\left|g_{\alpha}(x) f(x)-g(x) f(x)\right| \\
& \leqq\left\|g_{\alpha}-g\right\|_{K}\|f\|+\left\|g_{\alpha}-g\right\| \sup _{x \notin K}|f(x)| .
\end{aligned}
$$

The first term is small when $\alpha$ is large and the second term is small when $\varepsilon$ is small and $S$ is $\sigma$-bounded. Then $g \in B$ and hence $B=C$.

Recall that the Šilov boundary of a separating supremum norm algebra $A$ of continuous functions on a locally compact space $X$ is the 
smallest closed set of $X$ on which every function in $A$ assumes its maximal modulus. The Ślov boundary of $A$ in $X$ will be denoted by $\mathscr{C}(A ; X)$. The behaviors of the topologies $\sigma, \beta, \kappa$ on $M(A)$ are closely related to the topological properties of the Silov boundary $\mathscr{C}(A ; X)$.

THEOREM 4.4. Let $A$ be a supremum norm algebra. Then

$$
\mathscr{L}(M(A) ; X)=\mathscr{l}(A ; X) \text {. }
$$

Proof. Take $g \in M(A)$. Then $g f \in A$ whenever $f \in A$, and by 4.1 we have

$$
\begin{aligned}
\|g\| & =\sup _{\|f\|=1}\|g f\| \\
& =\sup _{\|f\|=1} \sup _{x \in \mathscr{M}^{(A ; X)}}|g(x) f(x)| \leqq \sup _{x \in \mathscr{M}^{(A ; X)}}|g(x)| .
\end{aligned}
$$

Hence

$$
\mathscr{\ell}(M(A) ; X) \subset \ldots \mathscr{l}(A ; X) \text {. }
$$

The reversed inequality is evident.

q.e.d.

Theorem 4.5. For the multiplier algebra $M(A)$ of a supremum norm algebra $A$, the topologies $\sigma$ and $\beta$ coincide if and only if $\mathscr{l l}(A ; X)$ is compact.

Proof. If $\mathscr{C}(A ; X)$ is compact, the topology $\kappa$ is finer than the topology $\sigma$. Comparing this result to Theorem 4.2 , we see that if $\mathscr{C l}(A ; X)$ is compact, then $\sigma=\beta=\kappa$.

Conversely suppose that $\sigma=\beta$. Then there exist $f_{1}, f_{2}, \cdots, f_{n} \in A$ and $\varepsilon>0$ such that for all $g \in M(A)$,

$$
\left\|g f_{i}\right\|<\varepsilon, \quad i=1,2, \cdots, n,
$$

imply $\|g\|<1$. As each $f_{i}$ vanishes at infinity, the set

$$
K=\bigcup_{i=1}^{n}\left\{x:\left|f_{i}(x)\right| \geqq \varepsilon\right\}
$$

is compact. Now suppose that $\mathscr{l}(A ; X)$ is not compact. Then there is a function $g \in A \subset M(A)$ such that $\|g\|_{K}<1$, and $\|g\|=g\left(x_{0}\right)=1$ for some $x_{0} \in \mathscr{l}(A ; X) \backslash K$. By raising $g$ to a sufficiently high power, we may assume without loss of generality that

$$
\|g\|_{K}<\frac{\varepsilon}{\sup _{i}\left\|f_{i}\right\|+1} .
$$

Then $\left|g(x) f_{i}(x)\right|<\varepsilon$ for all $x \in \mathscr{l}(A ; X)$. Thus $\left\|g f_{i}\right\|<\varepsilon$ and 
$\|g\|=1$. This contradicts our choice of $\varepsilon$. Hence $\mathscr{L}(A ; X)$ must be compact.

q.e.d.

Examining our proof again, we see that even more has been proved; namely, for a supremum norm algebra $A, \mathscr{M}(A ; X)$ is compact if and only if the topology on $A$ induced by $\beta$ coincides with the norm topology.

Obviously the condition that $\mathscr{C}(A ; X)$ is compact is also necessary and sufficient in order that $\sigma=\kappa$. We would also like to get topological conditions on $\mathscr{C}(A ; X)$ so that $\beta=\kappa$. We call a topological space $X$ countably precompact if $X$ is locally compact and Hausdorff, and if every countable union of compact sets in $X$ has a compact closure. We conjecture that $\beta=\kappa$ if and only if $\mathscr{C}(A ; X)$ is countably precompact. The following considerations support this conjecture:

THEOREM 4.6. Let $A$ be a supremum norm algebra with maximal ideal space $X$. If the restriction of every function in $A$ to $\mathscr{l}(A ; X)$ has a compact support, then the topologies $\beta$ and $\kappa$ for $M(A)$ coincide.

Proof. Let $\left\{g_{\alpha}\right\}$ be a net in $M(A) \kappa$-converging to a function $g \in M(A)$. We have only to show that $\left\{g_{\alpha}\right\}$ also converges to $g$ in the topology $\beta$. Thus take $f \in A$ and let $K$ be the support of the restriction of $f$ to $\mathscr{C l}(A ; X)$. By assumption, $K$ is compact. Thus the net $\left\{g_{\alpha} f\right\}$ converges uniformly on $K$, hence also uniformly on $X$, to $g f$. Thus $\beta=\kappa$.

For $f \in A$, the set $\{x: f(x) \neq 0\}$ is always a $\sigma$-compact set. This proves the following corollary:

COROLlaRY 4.7. Let $A$ be a supremum norm algebra with maximal ideal space $X$. If $\mathscr{C l}(A ; X)$ is countably precompact, then the topologies $\beta$ and $\kappa$ coincide.

Our conjecture can be proved in the following two special cases:

THEOREM 4.8. Let $X$ be a locally compact Hausdorff space and let $A$ be the supremum norm algebra of all complex-valued continuous functions on $X$ which vanish at infinity. In this case $\mathscr{L}(A ; X)=X$ and $M(A)$ is the algebra of all bounded continuous functions on $X$. Then the following conditions are equivalent:

(i) the topologies $\beta$ and $\kappa$ are equivalent.

(ii) every function in $A$ has a compact support.

(iii) $X$ is countably precompact

Proof. Suppose that (ii) does not hold, and let $f \in A$ be a function with a non-compact support. For any compact set $K$ there is a point 
$x_{K} \notin K$ where $f$ does not vanish. Define a function $g_{K}$ such that $g_{K} \in A$, $g_{K}(x)=1$ for $x \in K$ and

$$
g_{K}\left(x_{K}\right)>\frac{2\|f\|}{\left|f\left(x_{K}\right)\right|} .
$$

Then the net $\left\{g_{K}\right\}$ ordered by inclusion of the compact set $K$ converges in the topology $\kappa$ to the constant 1 . But

$$
\left\|g_{K} f-f\right\| \geqq\left|g_{K}\left(x_{K}\right)\right| \cdot\left|f\left(x_{K}\right)\right|-\|f\|>\|f\| .
$$

Hence $\left\{g_{K}\right\}$ does not converge strictly. This shows that (i) implies (ii).

That (ii) implies (iii) is evident and that (iii) implies (i) is a special case of Corollary 4.7.

THeOREM 4.9. Let $A$ be a supremum norm algebra with a $\sigma$-compact maximal ideal space $X$. Then the topologies $\beta$ and $\kappa$ for $M(A)$ coincide if and only if $\mathscr{C l}(A ; X)$ is compact.

Proof. We have only to show that if $\mathscr{C}(A ; X)$ is not compact, then $\beta \neq \kappa$. As $X$ is $\sigma$-compact, there are compact sets $K_{1} \subset K_{2} \subset \ldots$ such that $X=\bigcup_{n=1}^{\infty} K_{n}$. Without loss of generality we can choose these sets in such a way that each $K_{n}$ is included in the interior of $K_{n+1}$. As $\mathscr{C l}(A ; X)$ is not compact, for each $n$ there is a function $h_{n} \in A$ such that $\left\|h_{n}\right\|>\left\|h_{n}\right\|_{K_{n}}$. By raising $h_{n}$ to a sufficiently high power and then multiplying the resulting function with a suitable constant, we obtain a function $f_{n}$ such that $\left\|f_{n}\right\|=n$ and $\left\|f_{n}\right\|_{K_{n}}<(1 / n)$. Thus $\left\{f_{n}\right\}$ is an unbounded $\kappa$-Cauchy sequence. It cannot converge in the topology $\kappa$ to a bounded function. As $\beta$ is complete, we see that $\kappa$ is different from $\beta$.

Let us return to Theorem 4.8. In general the topology $\kappa$ for $M(A)$ is not complete. The completion of $M(A)$ with respect to $\kappa$ is the algebra of all continuous functions on $X$. Hence $\kappa$ is complete if and only if $X$ is pseudocompact. (For the definition and basic properties of pseudocompact spaces, see Glicksberg [4]). This fact together with Theorem 4.8 shows that every countably precompact space is pseudocompact. The converse of this statement is in general false, as the following example shows:

ExAmple. There is a pseudocompact space which is not countably precompact.

Construction. Let $\omega$ be the set of all ordinals up to and including the first infinite one and let $\Omega$ be the set of all ordinals up to but excluding the first uncountable one, both endowed with the order topology. 
Define $X=\omega \times \Omega$. It is obvious that $X$ is locally compact and Hausdorff. By a theorem of Henriksen and Isbell [6], $X$ is pseudocompact.

Let $A$ be the algebra of all continuous functions on $X$ which vanish at infinity. Using Theorem 4.8 , to show that $X$ is not countably precompact, we have only to show that $\beta \neq \kappa$ for the algebra $M(A)$.

For each $\alpha \in \Omega$ define the function $g_{\alpha} \in M(A)$ by

$$
g_{\alpha}(n, \beta)=\left\{\begin{array}{l}
n \text { if } n \text { is finite and } \beta=\alpha \text { is not a limit ordinal; } \\
0 \text { if otherwise. }
\end{array}\right.
$$

When $\Omega$ is naturally ordered, the net $\left\{g_{\alpha}\right\} \kappa$-converges to 0 . Now define a function $f$ on $X$ such that

$$
f(n, \beta)=\left\{\begin{array}{l}
1 / n \text { if } n \text { is finite and } \beta \text { is not a limit ordinal; } \\
0 \text { if otherwise. }
\end{array}\right.
$$

Then the net $\left\{g_{\alpha} f\right\}$ converges to 0 pointwise, but not strictly. Hence $\kappa \neq \beta$ for $M(A)$. Thus $X$ cannot be countably precompact.

In this example, both $\kappa$ and $\beta$ are complete topologies, and $\beta$ is strictly finer than $\kappa$. This shows the that the ordinary version of the open mapping theorem cannot be true for $\beta$.

5. Multipliers of supremum norm algebras-continued. In this section we collect together assorted results on supremum norm algebras. We start with

THeOREm 5.1. Let $A$ be a supremum norm algebra strictly dense in $M(A)$ and let $X$ be the maximal ideal space of $A$. If $g$ is a bounded function on $X$ such that $g A \subset M(A)$, then $g \in M(A)$.

Proof. By Theorem 2.4, $A$ has an approximate unit $\left\{f_{\alpha}\right\}$. Consider the net $\left\{f_{\alpha} g\right\}$. Since $g$ is assumed to be bounded $\left\{f_{\alpha} g\right\}$ is strictly Cauchy and converges to an element $h \in M(A)$. Since strict convergence implies pointwise convergence, we get $g=h \in M(A)$.

Next we consider measures on the Silov boundary to represent maximal ideals. Thus let $A$ be a supremum norm algebra with maximal ideal space $X$ and Šilov boundary $\mathscr{C}(A ; X)$. It is well-known that corresponding to each $x \in X$ there is a regular Borel measure $\mu_{x}$ on $\mathscr{C}(A ; X)$ with total mass 1 such that

$$
f(x)=\int_{\mathscr{B}^{\prime}(A ; X)} f(s) d \mu_{x}(s)
$$

for all $f \in A$. (See Arens and Singer [1]). This measure is not necessarily unique. We wish to find conditions under which one such measure exists such that 


$$
g(x)=\int_{\mathscr{M}^{(A ; X)}} g(s) d u_{x}(s)
$$

holds for all $g \in M(A)$.

THEOREM 5.2. The above formula holds for all $g \in M(A)$ if either of the following two conditions is satisfied:

(i) $\mathscr{C l}(A ; X)$ is compact;

(ii) there is a uniformly bounded sequence $\left\{f_{n}\right\} \subset A$ such that $\lim _{n \rightarrow \infty} f_{n}(x)=1$ for each $x \in X$.

Proof. (i) If $\mathscr{C}(A ; X)$ is compact, we may easily show that

$$
\mathscr{C l}(A ; X)=\mathscr{C l}(M(A) ; Y),
$$

where $Y$ is the maximal ideal space of $M(A)$. Select the measure $\mu_{x}$ according to the algebra $M(A)$, and the formula is trivially satisfied.

(ii) Let $\left\{f_{n}\right\}$ be the uniformly bounded "pointwise approximate unit" whose existence is assumed by the given condition. For $g \in M(A)$, we have $g f_{n} \in A$, and thus

$$
\int_{\mathscr{M}^{(A ; X)}} g(s) f_{n}(s) d \mu_{x}(s)=g(x) f_{n}(x)=g(x) \int f_{n}(s) d \mu_{x}(s)
$$

Let $n \rightarrow \infty$ and apply the Lebesgue bounded convergence theorem, we get

$$
g(x)=\int_{\mathscr{M}^{(A ; X)}} g(s) d \mu_{x}(s) . \quad \text { q.e.d. }
$$

Now we are going to consider a different class of "supremum norm algebras". Honestly they are not supremum norm algebra in the sense of the term hitherto used, and they occur here simply because no other place is more suitable for them.

Thus let $B$ be a given Banach algebra without order and let $\Omega$ be a compact Hausdorff space. We are going to consider the algebra $A=C(\Omega, B)$ of all continuous functions from $\Omega$ into $B$. If $f \in A$, define $\|f\|=\sup _{\omega \in \Omega}\|f(\omega)\|$. It is easy to prove that under this norm $A$ becomes a Banach algebra without order. $B$ can be isometrically embedded in $A$ if we identify an element $x$ in $B$ with the function which takes on the value $x$ identically. We want to find out what the multiplier algebra $M(A)$ is.

TheOREM 5.3. Using these notations, $M(A)=C(\Omega ; M(B))$, the algebra of all continuous functions from $\Omega$ into $M(B)$, where $M(B)$ is endowed with the strict topology. 
Proof. To show that $C(\Omega ; M(B)) \subset M(A)$, take $g \in C(\Omega ; M(B))$ and $f \in A$. Then for each $\omega \subseteq \Omega, g(\omega) f(\omega) \in B$. We have only to show that $g(\omega) f(\omega)$ is continuous in $\omega$. Thus let $\left\{\omega_{\alpha}\right\}$ be a net in $\Omega$ tending to $\omega_{0}$. Then $g\left(\omega_{\alpha}\right) \rightarrow g\left(\omega_{0}\right)$ strictly, and $f\left(\omega_{\alpha}\right) \rightarrow f\left(\omega_{0}\right)$ in norm. By the uniform boundedness principle, there is a constant $K$ such that $\left\|g\left(\omega_{\alpha}\right) x\right\|<K\|x\|$ for all $\alpha$ and all $x \in B$. Thus

$$
\begin{aligned}
\| g\left(\omega_{\alpha}\right) f\left(\omega_{\alpha}\right) & -g\left(\omega_{0}\right) f\left(\omega_{0}\right) \| \\
& \leqq K\left\|f\left(\omega_{\alpha}\right)-f\left(\omega_{0}\right)\right\|+\left\|\left[g\left(\omega_{\alpha}\right)-g\left(\omega_{0}\right)\right] f\left(\omega_{0}\right)\right\| \rightarrow 0 .
\end{aligned}
$$

Hence $g f \in A$.

Conversely let $T \in M(A)$. Take $f_{1}, f_{2} \in A$ such that $f_{1}(\omega)=f_{2}(\omega)$ for some $\omega \in \Omega$. We want to show that $\left(T f_{1}\right)(\omega)=\left(T f_{2}\right)(\omega)$. Suppose not, since $B$ is without order, there is an element $y \in B$ such that

$$
\begin{aligned}
0 \neq\left[\left(T f_{1}\right)(\omega)\right. & \left.-\left(T f_{2}\right)(\omega)\right] y \\
& =\left[T\left(f_{1}-f_{2}\right) y\right](\omega)=(T y)\left[f_{1}(\omega)-f_{2}(\omega)\right]=0 .
\end{aligned}
$$

This is a contradiction. As $B$ can be embedded in $A$, every value of $B$ is assumed at every point of $\Omega$ by some function in $A$. Thus $T f=g f$ for some function $g: \Omega \rightarrow M(B)$.

We still have to show that $g$ is continuous. Let $\left\{\omega_{\alpha}\right\} \subset \Omega$ be a net converging to a point $\omega_{0}$. Since for all $x \in B, g(\omega) x$ is a continuous function of $\omega$, we see that $g\left(\omega_{\alpha}\right) x \rightarrow g\left(\omega_{0}\right) x$. As this holds for every $x \in B, g\left(\omega_{\alpha}\right) \rightarrow g\left(\omega_{0}\right)$ in the strict topology of $M(B)$. This proves the continuity.

q.e.d.

6. Multipliers of semi-groups of characters. Let $G$ be a locally compact abelian group and let $X$ be the character group of $G$, both written additively. Then by a semi-group of characters of $G$ is meant a subset $S \subset X$ which is closed under addition. Let $S$ be a semi-group of characters of $G$. Then we define the multipliers of $S$ to be those elements $\chi \in X$ which satisfy $\chi+S \subset S$. The set $M(S)$ of all multipliers of $S$ evidently forms a semi-group of characters containing $S$ as an ideal. If $0 \in S$, then $M(S)=S$. Also if $S$ closed, so is $M(S)$. In this section we will study how the multiplier semi-group and the multiplier algebra are connected. The special case where $G$ is the circle group was mentioned in $\S 1$. As noted there, the tool for our proof is Fejér's theorem on the Cesáro summability. Hence in order to get a generalized version we look for a generalization of Fejér's theorem.

Fortunately such a generalization exists for compact groups $G$. It can be formulated as follows: There exists a net $\left\{\left(\chi_{1}^{\alpha}, \cdots, \chi_{N}^{\alpha}\right): \alpha \in A\right\}$ of finite sequences of characters and a net $\left\{\left(p_{1}^{\alpha}, \cdots, p_{N}^{\alpha}\right)\right\}$ of finite sequences of complex numbers such that for each continuous function $f$ on $G$ the net 


$$
\left\{\sum_{i=1}^{N \alpha} p_{i}^{\alpha}<f, \chi_{i}^{\alpha}>\chi_{i}^{\alpha}: \alpha \in A\right\}
$$

where

$$
\left\langle f, \chi_{i}^{\alpha}\right\rangle=\int f(t) \overline{\chi_{i}^{\alpha}(t)} d \mu(t),
$$

$\mu$ being the Haar measure, converges to $f$ uniformly. (For proof, see Silov [9]).

LEMmA 6.1. Let $S$ be a set of characters of a compact group $G$. Then the set of all continuous functions on $G$ uniformly approximable by finite linear combinations of characters $\chi \in S$ is identical with the set of all continuous functions whose Fourier series involve $\chi \in S$ only.

Proof. Denote the two sets under consideration by $A$ and $B$ respectively. By the above-mentioned generalization of Fejér's theorem, we have $B \subset A$. As every finite linear combination of elements of $S$ lies in $B$, we see that $B$ is dense in $A$. We have only to show that $B$ is uniformly closed. Thus let $\left\{f_{n}\right\}$ be a sequence in $B$ converging uniformly to a function $f$. Then $f$ is continuous, and

$$
\left\langle f_{n}, \chi\right\rangle \rightarrow\langle f, \chi\rangle
$$

for each character $\chi$. As $\left\langle f_{n}, \chi\right\rangle=0$ if $\chi \notin S$, we see that $f \in B$. This finishes the proof. q.e.d.

Now we come to the theorem announced before.

THEOREM 6.2. Let $S$ be a semi-group of characters of a compact group $G$ and let $M(S)$ be the multiplier semi-group of $S$. Let $A$ and $B$ be the algebras of all functions on $G$ uniformly approximable by finite linear combinations of characters in $S$ and $M(S)$ respectively. Then $B=M(A)$.

Proof. Evidently $M(S) \subset M(A)$. Hence $B \subset M(A)$, since $M(A)$ is uniformly closed.

Conversely suppose that $g \in M(A)$. We want to show that $g \in B$. Suppose that $\chi$ is a character not in $M(S)$. Then there is a character $\varphi \in S$ such that $\chi+\varphi \notin S$. Let $f=g \varphi$. Then $f \in A$ and $\langle f, \chi+\varphi\rangle=0$. Now

$$
\begin{aligned}
\langle f, \chi+\varphi\rangle & =\int f(t) \overline{\chi(t) \varphi(t)} d \mu(t) \\
& =\int g(t) \varphi(t) \overline{\chi(t) \varphi(t)} d \mu(t) \\
& =\int g(t) \overline{\chi(t)} d \mu(t)=\langle g, \chi\rangle .
\end{aligned}
$$


Hence $\langle g, \chi\rangle=0$. Thus by Lemma $6.1, g \in B$. q.e.d.

Considering the almost periodic compactification, this theorem can also be formulated as a theorem on the almost periodic functions on a locally compact group. Compare e.g., Loomis [8, Ch. VIII]. There is also a generalization of this theorem to locally compact groups $G$, and the special case where $G$ is compact provides an alternative proof of Theorem 6.2. First we define the spectrum of a continuous function $f$ on $G$ as follows: It will be a subset $\sigma(f)$ of the character group $X$ of G. A point $\chi_{0} \in X \mid \sigma(f)$ if and only if $\chi_{0}$ has a neighborhood $V$ such that

$$
\int \overline{\chi(f)} \chi(g) d \nu(\chi)=0
$$

for all functions $g \in L^{1}(G)$ the support of whose Fourier transform lies in $V, \nu$ being the Haar measure of $X$. We will make use of the following facts about the spectra of functions (see [3]):

Lemma 6.3. The spectra of functions satisfy

$$
\begin{aligned}
& \text { (i) } \sigma(f g) \subset \overline{[\sigma(f)+\sigma(g)]} \text {; } \\
& \text { (ii) if } \chi \in X \text {, then } \sigma(\chi g)=\chi+\sigma(g) \text {. }
\end{aligned}
$$

Having this, we can now prove

THEOREM 6.4. Let $G$ be a locally compact abelian group with character group $X$. Let $S$ be a closed subsemi-group of $X$. Further let $A$ and $B$ be the linear subspaces of all bounded continuous function on $G$ whose spectra lie in $S$ and $M(S)$ respectively. Then

$$
B=M(A) \text {. }
$$

Proof. If $g \in B$, then $\sigma(g) \subset M(S)$. For any $f \in A$,

$$
\sigma(g f) \subset \overline{[\sigma(g)+\sigma(f)]} \subset \overline{[M(S)+S\rceil} \subset S .
$$

Hence $g f \in A$, and $g \in M(A)$.

Conversely if $g \notin B$, then there exists a character $\chi$ in $\sigma(g) \backslash M(S)$. We can find a character $\varphi \in S \subset A$ such that $\chi+\phi \notin S$. Now

$$
\sigma(\varphi g)=\varphi+\sigma(g) .
$$

Hence $\chi \equiv \sigma(\phi g)$ and $\varphi g \notin A$. Thus $g \notin M(A)$.

q.e.d.

7. A list of some unsolved problems. As a postscript, we list here several unsolved problems.

(i) Give satisfactory descriptions of the closure of a Banach algebra $A$ without order in $M(A)$ with respect to the different topologies. 
(ii) Let $A$ be a *-algebra. Under what conditions is $M(A)$ also a *-algebra? Same problem for regular algebras.

(iii) Describe the closure of the maximal ideal space of a semisimple algebra $A$ in the maximal ideal space of $M(A)$.

(iv) Prove or disprove the conjecture mentioned before Theorem 4.6.

\section{BIBLIOGRAPHY}

1. R. Arens and I. M. Singer, Function values as boundary integrals, Proc. Amer. Math. Soc., 5 (1954), 735-745.

2. R. C. Buck, Bounded continuous functions on a locally compact space, Michigan Math. J., 5 (1958), 95-104.

3. K. deLeeuw and H. Mirkil, Translation invariant function algebras on Abelian groups, Bull. Soc. Math. France, 88 (1960), 345-370.

4. I. Glicksberg, The representation of functionals by integrals, Duke Math. J., 19 (1952). 254-261.

5. S. Helgason, Multipliers of Banach algebras, Ann. of Math., 64 (1956), 240-254.

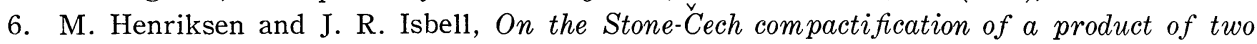
spaces, Bull. Amer. Math. Soc., 63 (1957), 145-146.

7. E. Hewitt, The asymmetry of certain algebras of Fourier-Stieltjes transforms, Michigan Math. J., 5 (1958), 149-158.

8. L. H. Loomis, An Introduction to Abstract Harmonic Analysis, New York, Van Nostrand, 1953.

9. G. E. Šilov, Homogeneous rings of functions, Amer. Math. Soc. Trans1., No. 92 (1953). 10. J. H. Williamson, A theorem on algebras of measures on topological groups, Proc. Edinburgh Math. Soc., 11 (1959), 195-206. 
